\title{
Application Fuzzy Decision Tree Analysis for Prediction Condensate Gas Ratio: Case Study
}

\author{
Mohammad Ebadi \\ Department of Petroleum \\ Engineering, Science and \\ Research Branch, Islamic \\ Azad University, Tehran, IRAN
}

\author{
Mohammad Ali Ahmadi \\ Petroleum University of \\ Technology, Ahwaz Faculty of \\ Petroleum Engineering, \\ Kut Abdollah, Ahwaz, \\ P.O.BOX:63134 IRAN \\ Reza Askarinezhad \\ Petroleum University of \\ Technology, Ahwaz Faculty of \\ Petroleum Engineering, \\ Kut Abdollah, Ahwaz, \\ P.O.BOX:63134 IRAN
}

\author{
Shahab Gerami \\ IOR Research Institute, \\ National Iranian Oil Company, \\ Tehran, IRAN
}

\begin{abstract}
Condensate-to-gas ratio (CGR) plays a significant role in sales potential assessment of both gas and liquid, design of the required surface processing facilities, and reservoir characterization and modeling in gas-condensate reservoirs. Precise field and laboratory determination of the CGR is time and people intensive. Developing a rapid and inexpensive technique for accurate estimation of the CGR is inevitable. To tackle this issue, an intelligent model was proposed and the purpose of this paper is to illustrate how Fuzzy Decision Tree (FDT), which is an automatic method of generating fuzzy rules, can predict the condensate gas ratio, as a vital parameter in order to design the necessary wellhead production facilities in retrograde gas reservoir. As the FDT method is strongly based on applying widely and effectively the concept of ambiguity and furthermore, to do this project more accurately and less dependent on experts' knowledge, it was decided to gain from piecewise linear membership functions (MFs) whose parameters have automatically been dedicated through calculating a very special method of possibility density function (pdf). In conclusion, according to the calculated result, it is possible to exploit this method for CGR prediction field wide. The Fuzzy model was evaluated using the experimental data combined with some PVT data from the open literature. The model predictions were compared with the data from the actual field data and experimental runs. Results from this study indicate that the developed model can predict the CGR with good precision. The proposed model can serve as a reliable tool for quick and cheap but effective assessment of the CGR in absence of any adequate laboratory or field data.
\end{abstract}

\section{General Terms}

Fuzzy Logic, Artificial Intelligence

\section{Keywords}

Fuzzy Logic, Decision Tree, Condensate Gas Ratio, Membership Function, Fuzzy Rule

\section{INTRODUCTION}

Increasing the gas cost and exploration of this kind of reservoir [1] show that these reservoirs become more and more valuable. It because of lower environmental damage [2]. Moreover, condensate liquid components have been more valuable than the gas, because of easy transportation especially in the places far from gas market or transport system [3]. As a result, understanding nonlinear and complex behavior of gas condensate reservoir is very important and also its one of the most difficult, onerous and challenging problem in petroleum reservoir engineering $[4,5]$. On the other hand behavior of gas condensate depends on PVT properties [4].

In the gas condensate reservoir, condensate liquid build up near well when the pressure drop below the dew point pressure $[3,5,6,7,8,9]$. This problem called condensate blockage [3] reduces permeability in the place that condensate is formed $[6,8,10]$ because liquid has lower mobility than the gas and high liquid-to-gas ratio [7, 11]. Due to these specifications some of the liquid in the reservoir is lost [11]. As a result it effects on productivity index because of accumulation of the condensate $[11,12]$. Consequently, prediction of gas condensate reservoir is very important.

Estimate the initial in place reserves and recovery factor are essential $[12,13]$ and these parameter depend on the liquid gas ratio production. It is defined by gas condensate ratio (CGR). CGR is very important parameter in gas condensate reservoir because condition of phases can be predict, the quality and quantity of facility can be designed, the economy of the reservoir can be predict, it can help numerical modeling, recovery factor and gas in place (GIP) can be estimated and etc. [1, 3, 14]

CGR depends on some factors [14]

$\begin{array}{ll}- & \text { Gas composition } \\ \text { - } & \text { Pressure } \\ \text { - } & \text { Temperature } \\ \text { API gravity } & \\ \text { undergoing equilibration } & \text { Relative quantity of oil and gas }\end{array}$


CGR can be calculate using three method; experimental data, equation of states and correlations $[15,16,17]$.

By using PVT tests, CGR can be measured. But we need sample of the reservoir. Sampling of the gas condensate reservoir has its problems. As a result obtain CGR from experimental data is expensive, complex, energy and time consuming, in errors, unavailable all time, inability to obtain representative sample and etc. $[17,18,19,14]$

$>\quad$ Equation of state need calibration from experimental data $[14,15]$ and it is not a good prediction measurement.

$>\quad$ Correlation cannot use in general cases and each field has its specific correlation. More over temperature in constant component cannot duplicate in this case. As a result these correlation should be obtain and test for each petroleum component. [4].

\section{METHODOLOGY}

To describe problematic issues in the current world where we do live in, we need to challenge with not exact, ambiguous and vague information [20]. As a result, Fuzzy Logic (FL) theory was primarily introduced by Lotfi A. Zadeh to defeat probable obstacles [21]. In more details, FL is an extended version of Boolean logic in which members of each set is dedicated by a unique degree [22]. In other words, objects are supposed to be a member of a set to some degree. It uses the spectrum of logical values between 0 (completely false) and 1 (completely true) [23]. There are a numerous number of distinguished models of Membership Functions (MFs) whose parameters can be determined either automatically or by experts. In this study, to do prevent any misunderstanding, it has been decided to utilize an automatic MF generator, proposed in [24], which gains from the benefits of a special method of Possibility Distribution Functions (pdf), referring to pdf functions as a method of MFs designing has been a topic of some relevant discussions since FL was proposed [25], which is based on Parzen window and related research [26-28].

$p d f_{j}(x)$

$=\frac{1}{\sqrt{2 m_{j} \pi}\left(\max \left(I_{j}\right)-\min \left(I_{j}\right)\right)} \sum_{i=1}^{m_{j}} \exp \left[-\frac{m_{j}}{2}\left(\frac{x-x_{i}}{\max \left(I_{j}\right)-\min \left(I_{j}\right)}\right)^{2}\right]$

In order to develop MFs, 158 series of gathered data (214 series) were randomly selected and the other series remained to test the model. Mfs of CGR and its affecting parameters, $\mathrm{Pd}, \mathrm{T}$ and $\mathrm{MW}$, based on the discussed technique are as below:

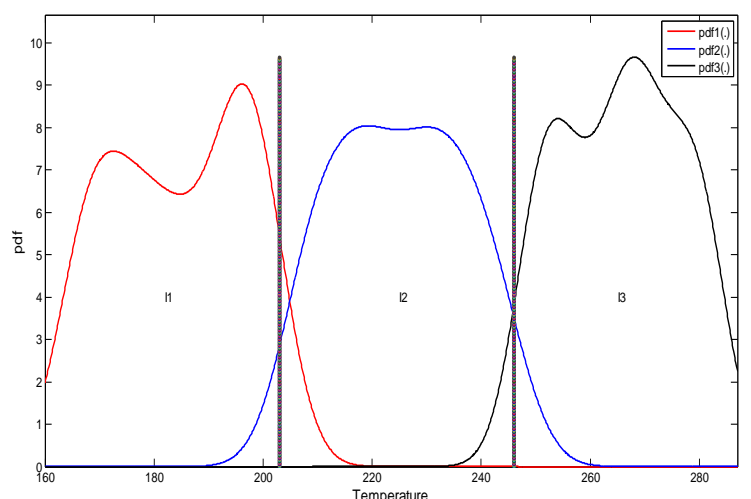

Figure 1: Estimated distribution related to intervals I1, I2, I3 for Temperature.

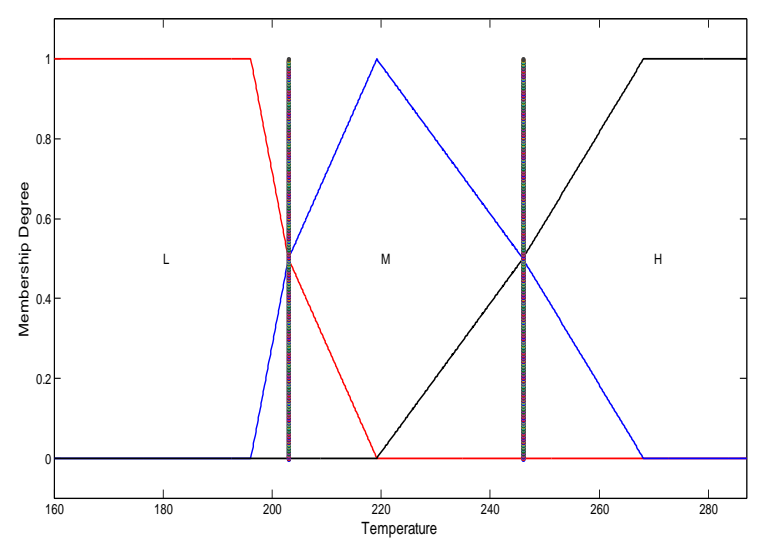

Figure 2: Membership functions of the decision classes L, $M$, and $H$ connected with Temperature.

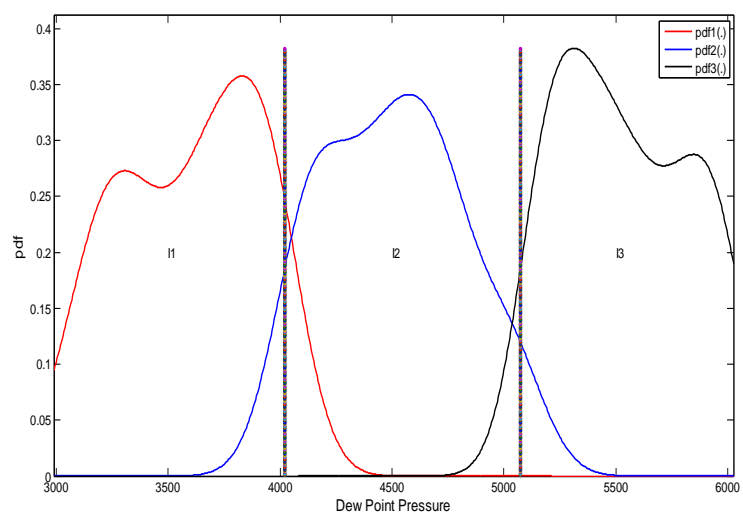

Figure 3: Estimated distribution related to intervals I1, I2, I3 for Dew Point Pressure. 


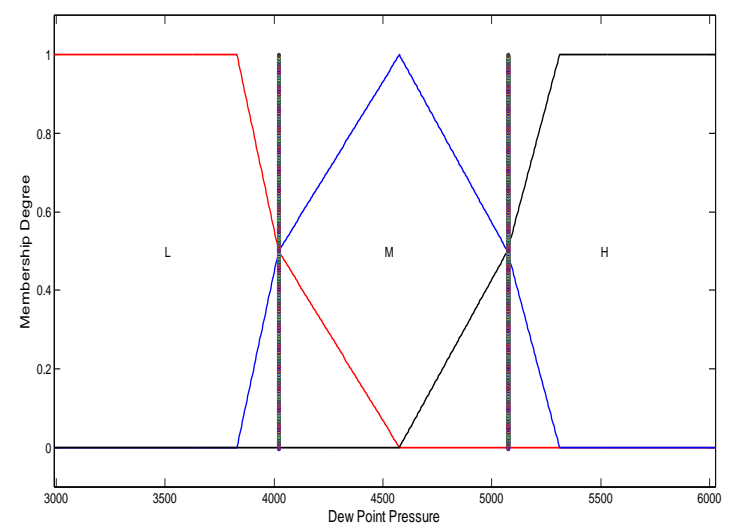

Figure 4: Membership functions of the decision classes $L$, $M$, and H connected with Dew Point Pressure.

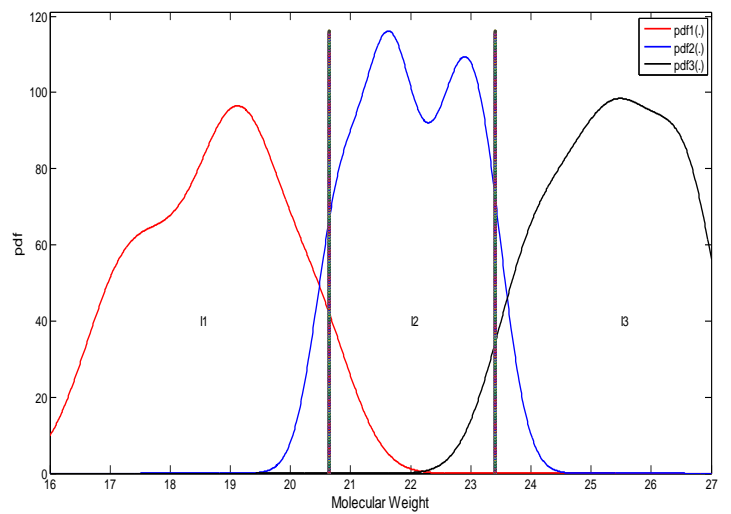

Figure 5: Estimated distribution related to intervals I1, I2, I3 for MolecularWeight.

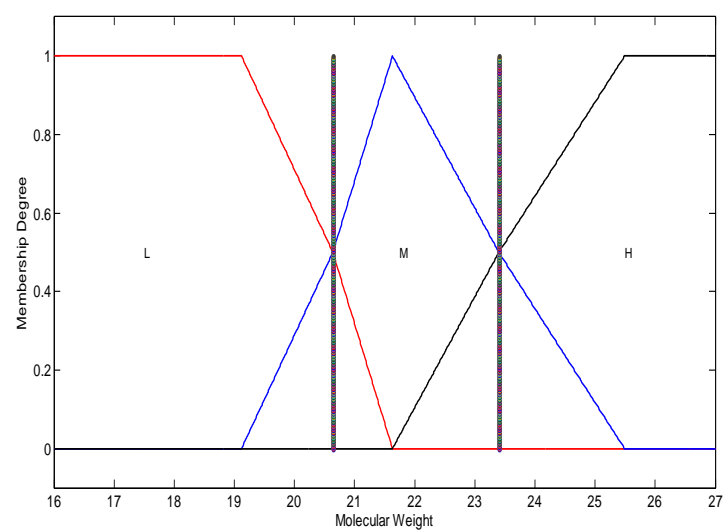

Figure 6: Membership functions of the decision classes $L$, $M$, and $H$ connected with Molecular Weight.

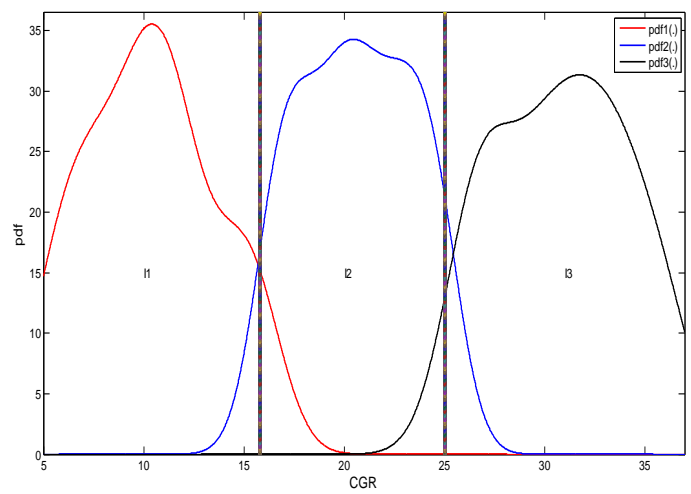

Figure 7: Estimated distribution related to intervals I1, I2, I3 for CGR.

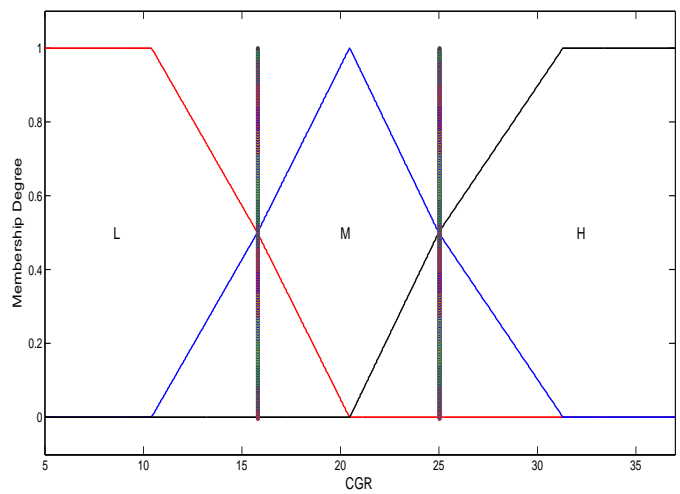

Figure 8: Membership functions of the decision classes L, $M$, and $H$ connected with CGR.

To conclude a CGR model as an extremely needed, industrial and concerning issue in upstream oil connected industries from these data, determined by their membership degrees, generating classification rules with linguistic terms is highly demanded to design efficiently wellhead facilities and equipment. Furthermore, it has been considered that a RBS can be paid attention as a remedy for vital, essential and critical reservoir engineers' challenging problems which most of them can implicitly be interpreted as a result of not having a precise model of CGR predicting. To construct fuzzy rules there are 3 probable methods [29] which are (1) From Human Experts: (2) Through literature Survey and (3) Automatic Rule Generation. To follow automatically this part of work and produce required rules there are some distinguished and well known techniques [30-32]. FDT is one of recent and cutting edge methods to generate automatically the fuzzy rules.

- Description of the inductive fuzzy decision tree method

Applying this method, results in generating series of fuzzy rules which characterize in linguistic terms. Its benefits can be summarized in measuring cognitive uncertainties and minimizing classification ambiguity when fuzzy evidences are present [33-34]. A membership function $\mu(\mathrm{x})$ of a fuzzy variable $\mathrm{Y}$ defined on $\mathrm{X}$, can be assumed as a possibility distribution of $\mathrm{Y}$ on $\mathrm{X}$, that is $\pi(\mathrm{x})=\mu(\mathrm{x})$, for all $\mathrm{x} \in \mathrm{X}$. The possibility measure $\mathrm{E} \alpha(\mathrm{Y})$ of ambiguity is defined as 
$E_{\alpha}(Y)=g(\pi)=\sum_{i=1}^{n}\left(\pi_{i}^{*}-\pi_{i+1}^{*}\right) \ln i$,

where $\pi^{*}=\left\{\pi_{1}^{*}, \pi_{2}^{*}, \ldots, \pi_{n}^{*}\right\}$ is the permutation of the possibility distribution $\pi=\left\{\pi\left(x_{1}\right), \pi\left(x_{2}\right), \ldots, \pi\left(x_{n}\right)\right\}$ sorted so that $\pi_{i}^{*} \geq \pi_{i+1}^{*}$ for $i=1, \ldots, n$, and $\pi_{n+1}^{*}=0$ [35]. The ambiguity of attribute $\mathrm{A}$ is then calculated as

$E_{\alpha}(A)=\frac{1}{m} \sum_{i=1}^{m} E_{\alpha}\left(A\left(u_{i}\right)\right)$

where $\mathrm{E}_{\alpha}\left(\mathrm{A}\left(\mathrm{u}_{\mathrm{i}}\right)\right)=\mathrm{g}\left(\mu \mathrm{T}_{\mathrm{s}}\left(\mathrm{u}_{\mathrm{i}}\right) / \max _{1 \leq \mathrm{j} \leq \mathrm{s}}\left(\mu \mathrm{T}_{\mathrm{j}}\left(\mu_{\mathrm{i}}\right)\right)\right)$, with $\mathrm{T}_{\mathrm{j}}$ the linguistic scale used within an attribute for $\mathrm{m}$ cases. Overlapping exist when there is overlapping between linguistic terms. The degree which $\mathrm{A}$ is a subset of $\mathrm{B}$ is measured through fuzzy subset hood S(A,B) and is given by [17]

$S(A, B)=\frac{\sum_{u \in U} \min \left(\mu_{A}(u), \mu_{B}(u)\right)}{\sum_{u \in U} \mu_{A}(u)}$,

Which all attributes are over the same set of objects.

The possibility of classifying an object to class $\mathrm{Ci}$, according to the given fuzzy evidence $\mathrm{E}$ can be defined as

$\pi=\left(C_{i} \mid E\right)=\frac{S\left(E, C_{i}\right)}{\max _{j} S\left(E, C_{j}\right)}$,

Where $S\left(E, C_{i}\right)$ performs the degree of truth for the classification rule (that is $\mathrm{E} \Rightarrow \mathrm{Ci}$ ). The classification ambiguity based on a single piece of evidence (a fuzzy value for an attribute) is defined as

$G(E)=g(\pi(C \mid E))$

$\mathrm{G}(\mathrm{P} \mid \mathrm{F})$ is the classification ambiguity with fuzzy partitioning $\mathrm{P}=\left\{\mathrm{E}_{1}, \ldots, \mathrm{E}_{\mathrm{k}}\right\}$ on the fuzzy evidence $\mathrm{F}$ which is the weighted average of classification ambiguity with each subset of partition:

$G(P \mid F)=\sum_{i=1}^{k} w\left(E_{i} \mid F\right) G\left(E_{i} \cap F\right)$,

Where $G\left(E_{i} \cap F\right)$ is the classification ambiguity with fuzzy evidence $E_{i} \cap F$, and where $w\left(E_{i} \mid F\right)$ is the weight $(w(\cdot))$ which indicates the relative size of subset $E_{i} \cap F$ in $F$ :

$w\left(E_{i} \mid F\right)=\frac{\sum_{u \in U} \min \left(\mu_{E_{i}}(u), \mu_{F}(u)\right)}{\sum_{j=1}^{k}\left(\sum_{u \in U} \min \left(\mu_{E_{j}}(u), \mu_{F}(u)\right)\right)}$.

In short, depend on the lowest level of ambiguity attributes are assigned to nodes. A node is terminated (becomes a leaf node), if degree of subset hood (based on the intersection of the nodes from the root) is higher than a truth value $\beta$ [36-38].

\section{Results and Discussion}

In this paper it was decided to have 3 different classes for each of attributes, Low (L), Medium (M) and High (H). Moreover, through applying $\beta$ value equating with 0.7 and after calculating the mentioned method, the succeeding results obtained as shown below:

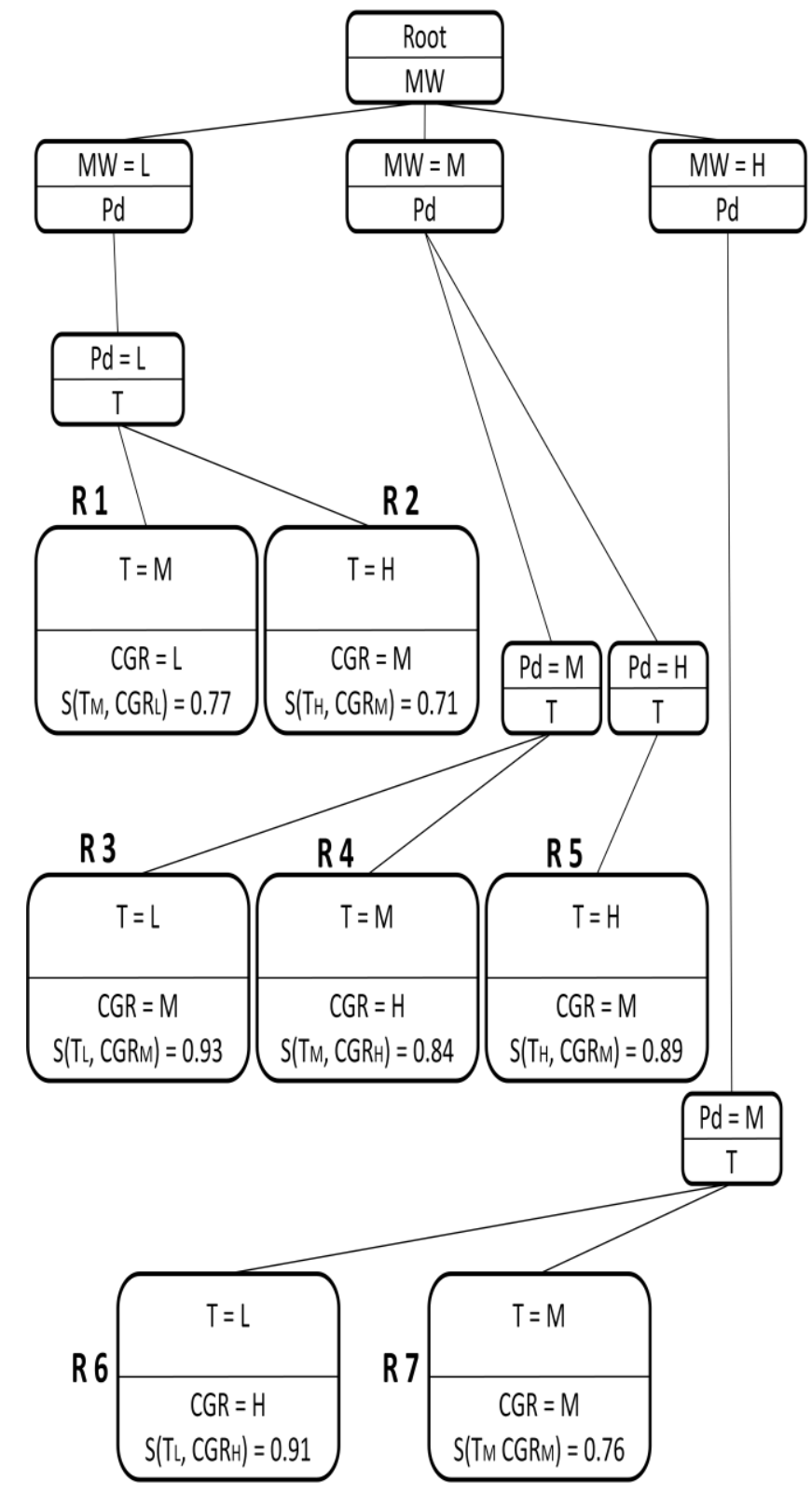

Figure 9: Schematic structure of fuzzy decision tree for CGR.

Table 1 : Class ambiguity values for each input attribute

\begin{tabular}{|c|c|c|c|}
\hline Attribute & MW & T & Pd \\
\hline $\mathrm{G}(\mathrm{E})$ & 0.248 & 0.7623 & 0.6324 \\
\hline
\end{tabular}

After finishing the process of constructing the FDT and extracting rules, 56 series of data in the set of testing were feed to the model and R-square of 0.631 was calculated for the responses. 


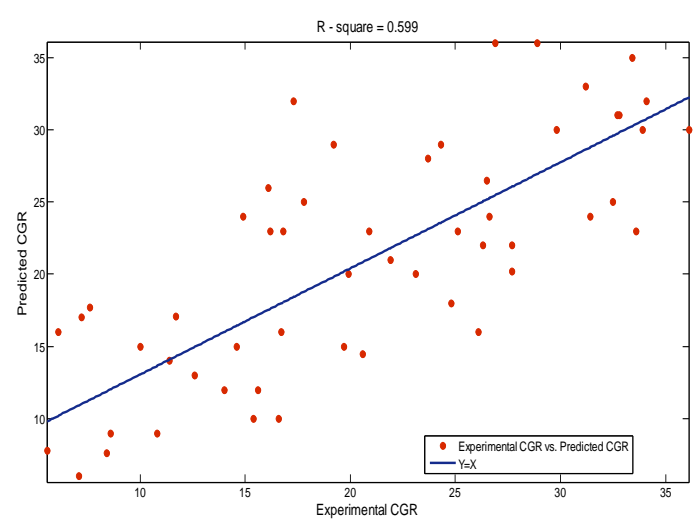

Figure 10: The performance of predicted values of CGR vs. the measured

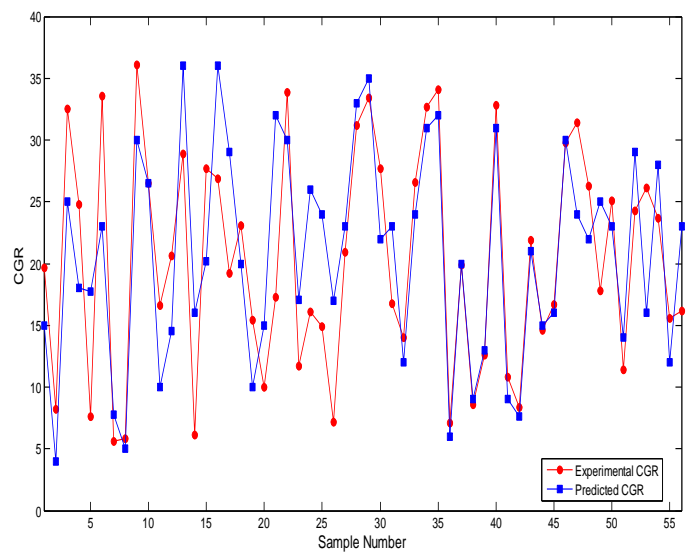

Figure 11: The comparison between predicted and measured values of CGR for each sample of testing set

\section{CONCLUSIONS}

\section{Based on results obtained from this work following conclusions can be drawn:}

However, it is acknowledged that in the current research the decision rules were restricted to three outcomes, identifying only low, medium and high fee levels. Clearly, if more intervals are specified more precision can be obtained with reference to the subsequent decision outcomes, depending on the particular application and the degree of clarity of linguistic interpretation required. Future research could examine the sensitivity of the results, by extending the analysis to petroleum applications, and in terms of increasing the classes (number of MFs) describing each attribute, together with the type of MF selected and the loss function associated with model errors.

This paper is concerned with the construction of a set of fuzzy rules using typical data reported in open literature and South pars gas reservoir. Central to the underlying philosophy towards this method has been the automation of the process to construct the fuzzy rules which limit the parameters which must be pre-specified by the expert (i.e. truth levels and number of membership functions).

\section{REFERENCES}

[1] Zhang H. R., Wheaton R. J., "Condensate Banking Dynamics in Gas Condensate Fields: Changes in Produced Condensate to Gas Ratios", International Oil and Gas Conference and Exhibition, China, Beijing, 7-10 November 2000.

[2] Orangi A., Nagarajan N. R., Honarpour M. M., Rosenzweig j., "Unconventional Shale Oil and GasCondensate Reservoir Production, Impact of Rock, Fluid, and Hydraulic Fractures", SPE Hydraulic Fracturing Technology Conference, USA, Texas, 24-26 January 2011.

[3] Fan Li, Harris Billy W., Jamaluddin A. J., Kamath Jairam, Mott Robert, Pope Gary A., Shandygin Alexander, Whitson Curtis Hays, "Understanding GasCondensate Reservoirs", Oilfield Review, 2005, 17(4), 14-27.

[4] Savary D., "Gas Condensate Reservoir Evaluation Using an Equation of State PVT Package", Middle East Oil Show, Bahrain, 16-19 November 1991.

[5] Eissa M., Shokri El-M., "Dewpoint Pressure Model for Gas Condensate Reservoirs Based on Genetic Programming", CIPC/SPE Gas Technology Symposium 2008 Joint Conference, Calgary, Alberta, Canada, 16-19 June 2008.

[6] Henderson, G.D., Danesh, A., Tehrani, D. H., Al-Shaidi, S., Peden, J.M., "Measurement and Correlation of Gas Condensate Relative Permeability by the Steady-State Method", SPE Reservoir Evaluation \& Engineering, 1998, 1(2), 134-140.

[7] Thomas F. B., Andersen G., Bennion D. B., "Gas Condensate Reservoir Performance", Journal of Canadian Petroleum Technology, July 2009, 48(7), 18 24.

[8] Luo Kai, Li Shi, Zheng Xitan, Chen Gang, Liu Ning, Sun Wenyue, "Experimental Investigation Into Revaporization of Retrograde Condensate", SPE Production and Operations Symposium, Oklahoma, Oklahoma City, March 2001.

[9] Ovalle Adriana P., Swaco M-I, Lenn Chris P., McCain Jr. William D., "Tools To Manage Gas/Condensate Reservoirs; Novel Fluid-Property Correlations on the Basis of Commonly Available Field Data", SPE Reservoir Evaluation \& Engineering, 2007, 10(6), 687694

[10] Alavi F.S., Mowla D., Esmaeilzadeh F., Journal of Canadian Petroleum Technology, 2010, 75(1-2), 44-53.

[11] Afidick, Deddy, Kaczorowski, N.J., "Production Performance of a Retrograde Gas Reservoir: A Case Study of the Arun Field", SPE Asia Pacific Oil and Gas Conference, Melbourne, Australia, 7-10 November 1994.

[12] Sadeghi Boogar A., Gerami S., Masihi M., "Investigation into the capability of a modern decline curve analysis for gas condensate reservoirs", Scientia Iranica, 2011, 18 (3), 491-501.

[13] Elsharkawy Adel M., "Predicting the dew point pressure for gas condensate reservoirs: empirical models and equations of state", Fluid Phase Equilibria, 2002, 193(12), 147-165 
[14] England W.A., "Empirical correlations to predict gas/gas condensate phase behaviour in sedimentary basins", 2002, 33(6), 665-673

[15] Nowroozi Saeed, Ranjbar Mohammad, Hashemipour Hasan, Schaffie Mahin, "Development of a neural fuzzy system for advanced prediction of dew point pressure in gas condensate reservoir", 2009, 90(3), 452-457

[16] Ovalle Adriana P., Swaco M-I, Lenn Chris P., McCain Jr. William D., "Tools To Manage Gas/Condensate Reservoirs; Novel Fluid-Property Correlations on the Basis of Commonly Available Field Data", SPE Reservoir Evaluation \& Engineering, 2007, 10(6), 687694

[17] M.Eissa, Shokir El-M., "Dew point Pressure Model for Gas Condensate Reservoirs Based on Genetic Programming”, Energy Fuels, 2008, 22(5), 3194-3200.

[18] Jamaluddin A.K.M., Dong C., Hermans P., Khan I.A., Carnegie A., Mullins O.C., Kurkjian A., Fujisawa G., Nighswander J., Babajan S., "Real-Time and On-Site Reservoir Fluid Characterisation using Spectral Analysis and PVT Express", APEA Journal, 44, 2004, 605-616.

[19] Humoud A.A., Al-Marhoun M.A., "A New Correlation for Gas-condensate Dewpoint Pressure Prediction”, SPE Middle East Oil Show, Bahrain, 17-20 March 2001.

[20] Ahmed Moataz A., Muzaffar Zeeshan, "Handeling Imprecision and Uncertainty in Software Development Effort Prediction: A Type-2 Fuzzy Logic Based Framework", Information and Software Technology, 2009, 51(3), 640-654

[21] Zadeh L. A., "Fuzzy Sets" Information and Control, $1965,8(3), 338-353$

[22] Ghafoori M. R., Roostaeian M., Sajjadian V. A., "A State-of-the-Art Permeability Modeling Using Fuzzy Logic in a Heterogeneous Carbonate (An Iranian Carbonate Reservoir Case Study)", International Petroleum Technology Conference, Kuala Lumpur, Malaysia, 3-5 December 2008.

[23] Hameed Ibrahim A., "Using Gaussian Membership Functions for Improving the Reliability and Robustness of Students' Evaluation Systems", Expert Systems with Applications, 38(6), 7135-7142.

[24] Beynon Malcolm J., Peel Michael J., Tang Yu-Cheng, "The Application of Fuzzy Decision Tree Analysis in an Exposition of the Antecedent of Audit Fees", Omega, 2004, 32(3) 231-244.

[25] Zadeh L. A., "Fuzzy Sets as a Basis for a Theory of Possibility", Fuzzy Sets and Systems, 1978, 1(1), 3-28.
[26] Parzen E., "On Estimation of a Probability Density Function Mode", Annals of Mathematical Statistics, 1962, 33(3), 1065-1076.

[27] Thompson JR, Tapia RA. Nonparametric function estimation, modeling, and simulation. Philadelphia: Society for Industrial and Applied Mathematics; 1990.

[28] Duda RO, Hart PE. Pattern classification and scene analysis. New York: Wiley; 1973.

[29] Kaleem Awan Malik Shahrzad, Awais Mina Muhammad, "Predicting Weather Events Using Fuzzy Rule Based System", Applied Soft Computing, 2011, 11(1), 56-63.

[30] Angelov P. P, Buswell R. A, "Automatic Generation of Fuzzy Rule-based Models from Data by Genetic Algorithms", Information Sciences, 2003, 150(1-2), 1731

[31] Wang Lian, Yen John, "Extracting fuzzy rules for system modeling using a hybrid of genetic algorithms and Kalman filter", Fuzzy Sets and Systems, 1999, 101(3), 353-362.

[32] Park Hui-June, Lim Jong-Se, Roh Jeongyong, Kang Joo M., Min Bae-Hyun, "Prediction-System Optimization of Gas Field Using Hybrid Fuzzy/Genetic Approach", SPE Journal, 2010, 15(2), 417-425.

[33] Liu Xiaodong, Pedrycz Witold, "The Development of Fuzzy Decision Tree in the Framework of Axiomatic Fuzzy Set Logic", Applied Soft Computing, 2007, 7(1), 325-342.

[34] Wang Xi-Zhao, Zhai Jun-Hai, Lu Shu-Xia, "Induction of Multiple Fuzzy Decision Trees Based on Rough Set Technique", Information Sciences, 2008, 178(16), 31883202.

[35] Higashi M, Klir GJ, "Measures of Uncertainty and Information Based on Possibility Distributions", International Journal of General Systems, 9(1), 43-58.

[36] Kosko Bart, "Fuzzy Entropy and Conditioning", Information Science, 1986, 40(2), 165-174.

[37] Yuan Yufei, Shaw Michael J., "Induction of Fuzzy Decision Trees", Fuzzy Sets and Systems, 1995, 69(2), 125-139.

[38] Wang Xizhao, Chen Bin, Qian Guoliang, Ye Feng, "On the Optimization of Fuzzy Decision Trees", Fuzzy Sets and Systems, 112(1), 117-125.

[39] Roa-Sepulveda C.A., Herrera M., Pavez-Lazo B., Knight U.G., Coonick A.H., "Economic Dispatch Using Fuzzy Decision Trees", Electric Power Systems Research, 2003, 66(2), 115-122. 